Pacific Journal of Mathematics

THE DESCENDING CHAIN CONDITION RELATIVE TO 


\title{
THE DESCENDING CHAIN CONDITION RELATIVE TO A TORSION THEORY
}

\author{
Robert W. Miller and Mark L. Teply
}

\begin{abstract}
A well-known theorem of Hopkins and Levitzki states that any left artinian ring with identity element is left noetherian. The main theorem of this paper generalizes this to the situation of a hereditary torsion theory with associated idempotent kernel functor $\sigma$. It is shown that if a ring $R$ with identity element has the descending chain condition on $\sigma$-closed left ideals, then $R$ has the ascending chain condition on $\sigma$-closed left ideals.
\end{abstract}

The remainder of the paper generalizes some results of Faith and Walker concerning artinian and quasi-Frobenius rings. In the case that the localization functor $\mathscr{L}_{\sigma}$ is exact the following are obtained: (1) a sufficient condition for the ring $R$ to have the descending chain condition on $\sigma$-closed left ideals and (2) characterizations of the condition that every $\sigma$-torsion-free injective left $R$ module is codivisible (projective).

In this paper $R$ always denotes ring with identity element, and unless denoted to the contrary, all modules are members of the category $R$-mod of unital left $R$-modules.

A subfunctor $\sigma$ of the identity functor on $R$-mod is called a left exact radical (or idempotent kernel functor) if $\sigma$ is left exact and $\sigma(M / \sigma(M))=0$ for every module $M$. Such a $\sigma$ naturally determines a torsion class $\mathscr{T}_{\sigma}=\{M \mid \sigma(M)=M\}$ and a torsion-free class $\mathscr{F}_{\sigma}=$ $\{M \mid \sigma(M)=0\}$. The pair $\left(\mathscr{\sigma}_{\sigma}, \mathscr{F}_{\sigma}\right)$ forms a hereditary torsion theory in the sense of [2], [10], [13], [14] and [15]. Then $\mathscr{T}_{\sigma}$ is closed under submodules, homomorphic images, direct sums, and extensions of one member of $\mathscr{T}_{\sigma}$ by another; and $\mathscr{F}_{\sigma}$ is closed under submodules, direct products, injective hulls, and extensions of one member of $\mathscr{F}_{0}$ by another. Also associated with $\sigma$ is the localization functor $\mathscr{L}_{\sigma}$ as defined in [2], [4], [13] or [14]. The module $\mathscr{L}_{\sigma}(R)$ can be made into ring by defining multiplication in a natural way; this ring will be denoted by $Q_{\sigma}$. A torsion theory is called perfect in [2], [12] and [13] if $\mathscr{L}_{\sigma}(M) \cong Q_{\sigma} \otimes_{R} M$ for every module $M$. For additional details on the concepts discussed in this paragraph, the reader is referred to [2], [4], [9], [10], [13], [14], and their references.

A submodule $N$ of $M$ is called $\sigma$-closed if $M / N \in \mathscr{F}_{\sigma}$. The lattice, of $\sigma$-closed submodules has been studied in [3], [5], [9], [12], [14], and [15]. Particular attention is usually given to chain conditions on $\sigma$-closed modules. We continue this investigation and focus our 
attension on the descending chain condition for $\sigma$-closed submodules of ${ }_{R} R$ (i.e., the $\sigma$-closed left ideal).

A well-known theorem of Hopkins [6] and Levitzki [8] states that any left artinian ring with identity element is left noetherian. Manocha [9] has generalized this result by showing that if $\sigma$ is perfect and if $R$ has the descending chain condition (dcc) on $\sigma$-closed left ideals, then $R$ has the ascending chain condition (acc) on $\sigma$-closed left ideals. The main result of the first section (Theorem 1.4) removes the very restrictive hypothesis that $\sigma$ is perfect from Manocha's result. Proofs of the result of Hopkins and Levitzki all seem to depend strongly on the nilpotence of the (Jacobson, or nil) radical; Manocha's proof uses the Hopkins-Levitzki result on $Q_{\sigma}$ and depends only on a lattice isomorphism between the $\sigma$-closed left ideals of $R$ and the left ideals of $Q_{\sigma}$ (a consequence of $\sigma$ being perfect). In our case where there is no restriction on $\sigma$, we can rely neither on nilpotence nor on a lattice isomorphism; our method of proof will depend on finding a substitute for actual nilpotence of the (Jacobson) radical and applying Goldman's results on modules of $\sigma$ finite length [5].

In the second section we generalize some results of Faith and Walker [1] to obtain a sufficient condition for $R$ to have dec on $\sigma$ closed left ideals when $\mathscr{L}_{\sigma}$ is exact. In particular, we show in Theorem 2.3 that if $\mathscr{L}_{\sigma}$ is exact and if each module in $\mathscr{F}_{\sigma}$ is contained in a direct sum of finitely generated modules, then $R$ has dec on $\sigma$-closed left ideals.

In the third section we apply the results of the first two sections to answer the following question in the case where $\mathscr{L}_{\sigma}$ is exact: For which $\sigma$ is every injective module in $\mathscr{F}_{\sigma}$ projective? Our answer to this question (given in Theorems 3.5 and 3.6) gives a generalization of an important theorem of Faith and Walker [1, Theorem 5.3] on quasi-Frobenius rings.

1. DCC implies ACC. In this section we show that if $R$ has dec on $\sigma$-closed left ideals, then $R$ also has acc on $\sigma$-closed left ideals. In order to do this we first recall two definitions from [2] and [3]. A nonzero module $M$ is $\sigma$-cocritical if $M \in \mathscr{F}_{0}$ and every proper homomorphic image of $M$ is in $\mathscr{T}_{\sigma}$. Nonzero submodules of $\sigma$ cocritical modules are $\sigma$-cocritical modules. If $M$ is a nonzero module in $\mathscr{F}_{\sigma}$ and if $M$ has dec on $\sigma$-closed submodules, then $M$ contains a $\sigma$-cocritical submodule. A submodule $N$ of a module $M$ is called $\sigma$ critical if $M / N$ is $\sigma$-cocritical. Thus a submodule $N$ of a module $M$ in $\mathscr{F}_{\sigma}$ is $\sigma$-critical if and only if $N$ is maximal among the proper $\sigma$-closed submodules of $M$. If there exist $\sigma$-cocritical modules, then there exist cyclic $\sigma$-cocritical modules; so we may define 


$$
V=\cap\{I \mid I \subseteq R, I \sigma \text {-critical }\} .
$$

Then $V$ is $\sigma$-closed, and $V$ is a proper two-sided ideal of $R$. If $N$ is a $\sigma$-cocritical module, then $V N=0$. We continue to use $V$ as a standard notation in this section.

Our first lemma is an analogue of the fact that, in a left artinian ring, the Jacobson radical is nilpotent.

Lemma 1.1. If $R$ has dec on $\sigma$-closed left ideals, then there exists a positive integer $n$ such that $V^{n+q} / V^{n+q+1} \in \mathscr{T}_{\sigma}$ for all $q \geqq 0$.

Proof. Suppose not. Then there exists a strictly increasing sequence $\left\{n_{i}\right\}$ of positive integers such that each $V^{n_{i}} / V^{n_{i}+1} \notin \mathscr{T}_{\sigma}$. Let $T_{n_{i}} / V^{n_{i}+1}=\sigma\left(V^{n_{i}} / V^{n_{i}+1}\right)$. Choose a left ideal $M_{i}$ of $R$ containing $T_{n_{i}}$ which is maximal with respect to the property that $M_{i} \cap V^{n_{i}}=T_{n_{i}}$. Via the natural map $R / T_{n_{i}} \rightarrow R / M_{i}$ we see that $V^{n_{i}} / T_{n_{i}} \in \mathscr{F}_{\sigma}$ is isomorphic to an essential submodule of $R / M_{i}$; hence $M_{i}$ is a $\sigma$-closed left ideal of $R$. For each positive integer $j$, let $N_{j}=\bigcap_{i=1}^{j} M_{i}$. Since intersections of $\sigma$-closed submodules are always $\sigma$-closed, then $N_{j}$ is $\sigma$-closed. Now $N_{j} \supseteqq T_{n_{j}} \supseteqq V^{n_{j+1}} \supseteqq V^{n_{j+1}}$. Furthermore $N_{j+1} \supseteqq V^{n_{j+1}}$; for if $V^{n_{i}} \cong N_{i} \subseteq M_{i}$, then $V^{n_{i}} / V^{n_{i}+1}=T_{n_{i}} / V^{n_{i}+1} \in \mathscr{T}_{o}$, which is contrary to the choice of the $n_{i}$ 's. Therefore, for each positive integer $j, N_{j} \neq N_{j+1}$, and we have an infinite, strictly descending chain $\left\{N_{i}\right\}$ of $\sigma$-closed left ideals of $R$. This contradicts our hypothesis that $R$ has dec on $\sigma$-closed left ideals.

In [5] a module $M$ is said to have $\sigma$-finite length if there exists a finite chain

$$
0=M_{n} \subset M_{n-1} \subset M_{n-2} \subset \cdots \subset M_{0}=M
$$

of submodules of $M$ such that $M_{i} / M_{i+1}$ is $\sigma$-cocritical for each $i=$ $0,1,2, \cdots, n-1$; we call the chain $\left(^{*}\right)$ a $\sigma$-composition series of $M$. In [5] it is shown that (1) any two $\sigma$-composition series of a module of $\sigma$-finite length have the same number of terms and that (2) a module $M$ has $\sigma$-finite length if and only if $M$ has both acc and dec on $\sigma$-closed submodules.

Our next lemma may be viewed as a specialization of [5, Proposition 2.10] and [3, Proposition 2.1(3)].

LEMMA 1.2. Let $M$ be a module for which 0 is an intersection of finitely many $\sigma$-critical submodules of $M$. Then there exist $\sigma$ cocritical submodules $N_{1}, N_{2}, \cdots, N_{k}$ of $M$ such that $\sum_{i=1}^{k} N_{i}$ is an essential direct submodule of $M$ and $M /\left(\sum_{i=1}^{k} N_{i}\right) \in \mathscr{T}_{\sigma}$.

Proof. By hypothesis $M$ is isomorphic to a submodule of a 
direct sum of finitely many $\sigma$-cocritical modules. Since this direct sum clearly has $\sigma$-finite length, then by [5, Corollary 1.5 and Proposition 1.2] $M$ has both acc and dec on $\sigma$-closed submodules. We now use induction to choose the desired modules $N_{i}$.

Since $M$ has dec on $\sigma$-closed submodules, we can choose a $\sigma$ cocritical submodule $N_{1}$ of $M$. Let $0 \neq x \in N_{1}$. There exists a $\sigma$ critical submodule $C_{1}$ such that $x \notin C_{1}$ by hypothesis. Now $0 \neq$ $N_{1} /\left(N_{1} \cap C_{1}\right) \cong\left(C_{1}+N_{1}\right) / C_{1} \in \mathscr{F}_{\sigma}$; so, since $N_{1}$ is $\sigma$-cocritical $N_{1} \cap C_{1}=0$. Suppose that $N_{1}, N_{2}, \cdots, N_{t}$ and $C_{1}, C_{2}, \cdots, C_{t}$ have been chosen such that $N_{i}$ is $\sigma$-cocritial, $C_{i}$ is $\sigma$-critical, $N_{i} \cong \bigcap_{j \leqq i-1} C_{j}$, and $N_{i} \cap C_{\imath}=0$ for each $i \leqq t$. If $\bigcap_{j=1}^{t} C_{j} \neq 0$, then we can choose $N_{t+1}$ to be a $\sigma$ cocritical submodule of $\bigcap_{j=1}^{t} C_{j}$. As in the discussion of case $N_{1}$, we can find a $\sigma$-critical submodule $C_{t+1}$ of $M$ such that $N_{t+1} \cap C_{t+1}=0$. Since $M$ has dcc on $\sigma$-closed submodules, there exists an integer $k$ such that $\bigcap_{j=1}^{k} C_{j}=0$; so the inductive process stops after $k$ steps. It follows from the construction that $\sum_{i=1}^{k} N_{i}$ is direct.

It remains to show that $M /\left(\sum_{i=1}^{k} N_{i}\right) \in \mathscr{T}_{\sigma}$. To do this it is sufficient to show by induction that $M /\left(\left(\sum_{i=1}^{t} N_{i}\right)+\left(\bigcap_{i=1}^{t} C_{i}\right)\right) \in \mathscr{T}_{\sigma}$ for each $t=1,2, \cdots, k$. Since $M / C_{1}$ is $\sigma$-cocritical and $\left(N_{1}+C_{1}\right) / C_{1} \neq 0$, then $M /\left(C_{1}+N_{1}\right) \in \mathscr{T}_{o}$; so the first case is established. We now assume that the result is true for all integers $<t$. Since $0 \neq$ $\left(N_{t}+C_{t}\right) / C_{t} \subseteq\left(\left(\bigcap_{i=1}^{t-1} C_{i}\right)+C_{t}\right) / C_{t}$ and $M / C_{t}$ is $\sigma$-cocritical, then

$$
\left(\left(\bigcap_{i=1}^{t-1} C_{i}\right)+C_{t}\right) /\left(N_{t}+C_{t}\right) \in \mathscr{S}_{\sigma}^{-} .
$$

Since $N_{t} \cong \bigcap_{i=1}^{t-1} C_{i}$, for each $x \in \bigcap_{i=1}^{t-1} C_{i}$ we obtain $\left(N_{t}+\bigcap_{i=1}^{t} C_{i}: x\right)=$ $\left(\left(\left(N_{t}+C_{t}\right) \cap\left(\bigcap_{i=1}^{t-1} C_{i}\right)\right): x\right)=\left(\left(N_{t}+C_{t}\right): x\right)$. Thus by $\left.{ }^{* *}\right)$ we obtain $\left(\bigcap_{i=1}^{t-1} C_{i}\right) /\left(N_{t}+\bigcap_{i=1}^{t} C_{i}\right) \in \mathscr{T}_{\sigma}$. Since $\mathscr{T}_{\sigma}$ is closed under homomorphic images, we have $\left(\left(\sum_{i=1}^{t-1} N_{i}\right)+\left(\bigcap_{i=1}^{t-1} C_{i}\right)\right) /\left(\left(\sum_{i=1}^{t} N_{i}\right)+\left(\bigcap_{i=1}^{t} C_{i}\right)\right) \in \mathscr{T}_{\sigma}$. Thus from the induction hypothesis and the exact sequence

$$
0 \longrightarrow \frac{\sum_{i=1}^{t-1} N_{i}+\bigcap_{i=1}^{t-1} C_{i}}{\sum_{i=1}^{t} N_{i}+\bigcap_{i=1}^{t} C_{i}} \longrightarrow \frac{M}{\sum_{i=1}^{t} N_{\imath}+\bigcap_{i=1}^{t} C_{i}} \longrightarrow \frac{M}{\sum_{i=1}^{t-1} N_{i}+\bigcap_{i=1}^{t-1} C_{\imath}} \longrightarrow 0
$$

we obtain $M /\left(\left(\sum_{i=1}^{t} N_{i}\right)+\left(\bigcap_{i=1}^{t} C_{i}\right)\right) \in \mathscr{T}_{\sigma}$ as desired.

As an immediate consequence of Lemma 1.2, we have the following analogue for $\sigma$ of the structure theorem for semisimple rings with dec.

CoROLlary 1.3. If $R$ has dec on $\sigma$-closed left ideals, then there exist $\sigma$-cocritical submodules $A_{1} / V, A_{2} / V, \cdots, A_{k} / V$ such that $\sum_{i=1}^{k}\left(A_{i} / V\right)$ is a direct essential submodule of $R / V$ and $(R / V) / \bigoplus_{i=1}^{k}\left(A_{i} / V\right) \in \mathscr{T}_{\sigma}$. 
We can now obtain the main result of this section.

TheOREm 1.4. Let $R$ have dcc on $\sigma$-closed left ideals. If a module $B$ has dec on $\sigma$-closed submodules, then $B$ also has acc on $\sigma$-closed submodules. In particular, $R$ has acc on $\sigma$-closed left ideals.

Proof. Let $B$ be a module with dec on $\sigma$-closed submodules. Let $I_{0}=\sigma(B)$. For $j \geqq 1$, define $I_{j}$ by $I_{j} / I_{j-1}$ is a minimal, nonzero, $\sigma$-closed submolde of $B / I_{j-1}$; such an $I_{j}$ exists whenever $0 \neq B / I_{j-1}$ (as $B / I_{j-1} \in \mathscr{F}_{\sigma}$ and has dec on $\sigma$-closed submodules). Moreover, $I_{j} / I_{j-1}$ is $\sigma$-cocritical by the minimality. It is sufficient to show that $I_{s}=B$ for some index $s$; for then $B / I_{0}$ has acc on $\sigma$-closed submodules by [5, Proposition 1.2], and hence $B$ has acc on $\sigma$-closed submodules (as the lattice of $\sigma$-closed submodules of $B / I_{0}$ is clearly isomorphic to the lattice of $\sigma$-closed submodules of $B$ ).

Assume for contradiction that $I_{j} \neq B$ for each $j \in Z^{+}$, which $Z^{+}$ denotes the set of positive integers. Set $m_{0}=0$, and let $m_{t+1}=\max \Gamma_{t}$, where $\Gamma_{t}=\left\{j \in Z^{+} \mid V x \subseteq I_{m_{t}}\right.$ for some $\left.x \in I_{j}-I_{j-1}\right\}$. Note that $m_{t}+1 \in \Gamma_{t}$ as $V\left(I_{m_{t}+1} / I_{m_{t}}\right)=0$. Inductively, assume that $m_{t}$ exists; we show via the next three paragraphs that $m_{t+1}$ exists.

Suppose not. Then for an infinite set $\Omega$ of indices $j>m_{t}+1$, we may choose $x_{j} \in I_{j}-I_{j-1}$ such that $V x_{j} \cong I_{m_{t}}$. By Corollary 1.3 $R / V$ contains an essential submodule of the form $\bigoplus_{i=1}^{k}\left(A_{i} / V\right)$, where each $A_{i} / V$ is $\sigma$-cocritical and $(R / V) / \bigoplus_{i=1}^{k}\left(A_{i} / V\right) \in \mathscr{T}_{\sigma}$. If for each $i=1,2, \cdots, k$ we have $A_{i} x_{j} \subseteq I_{m_{t}}$, then $\left(\sum_{i=1}^{k} A_{\imath}\right) x_{j} \subseteq I_{m_{t}} ;$ so $0 \neq$ $\left(R x_{j}+I_{m_{t}}\right) / I_{m_{t}} \in \mathscr{S}_{\sigma}$. But $B / I_{m_{t}} \in \mathscr{F}_{\sigma}$ by construction, which yields a contradiction. Thus for at least one of the $A_{i} / V, A_{i} x_{j} \nsubseteq I_{m_{t}}$.

Next assume that, for any such $A_{i} / V$ with $A_{i} x_{j} \nsubseteq I_{m_{t}}$, we have $\left(A_{i} x_{j}+I_{m_{t}}\right) \cap I_{j-1} \supsetneq I_{m_{t}}$. Since $\left(A_{i} x_{j}+I_{m_{t}}\right) / I_{m_{t}} \subseteq B / I_{m_{t}} \in \mathscr{F}_{\sigma}$ and $A_{i} / V$ is $\sigma$-cocritical, we see that the natural epimorphism $A_{i} / V \rightarrow$ $\left(A_{i} x_{j}+I_{m_{t}}\right) / I_{m_{t}}$ is an isomorphism. Thus $\left(A_{2} x_{j}+I_{m_{t}}\right) / I_{m_{t}}$ is $\sigma$-cocritical, and we have

$$
\begin{aligned}
\left(A_{i} x_{j}\right. & \left.+I_{m_{t}}\right) /\left(A_{i} x_{j}+I_{m_{t}}\right) \cap I_{j-1} \\
& \cong\left(\left(A_{i} x_{j}+I_{m_{t}}\right) / I_{m_{t}}\right) /\left(\left(\left(A_{i} x_{j}+I_{m_{t}}\right) \cap I_{j-1}\right) / I_{m_{t}}\right) \in \mathscr{T}_{\sigma}
\end{aligned}
$$

by assumption. But we also have $\left(A_{i} x_{j}+I_{m_{t}}\right) /\left(A_{i} x_{j}+I_{m_{t}}\right) \cap I_{j-1} \cong$ $\left(A_{i} x_{j}+I_{j-1}\right) / I_{j-1} \subseteq B / I_{j-1} \in \mathscr{F}_{\sigma}$. We conclude that $A_{i} x_{j} \subseteq I_{j-1}$ for any $A_{i} / V$ with $A_{i} x_{j} \nsubseteq I_{m_{t}}$. Now for each of the remaining $A_{i} / V$, we have $A_{i} x_{j} \subseteq I_{m_{t}} \subseteq I_{j-1}$. Hence $\left(\sum_{i=1}^{k} A_{j}\right) x_{j} \subseteq I_{j-1}$, which leads to a contradiction as $R / \sum_{i=1}^{k} A_{i} \in \mathscr{T}_{\sigma}$ and $B / I_{j-1} \in \mathscr{F}_{\sigma}$.

We have now established that, for each $j \in \Omega$, there exists a left ideal $A_{j}$ of $R$ and an $x_{j} \in I_{j}-I_{j-1}$ such that $A_{j} x_{j} \nsubseteq I_{m_{t}}$, 
$\left(A_{j} x_{j}+I_{m_{t}}\right) / I_{m_{t}}$ is $\sigma$-cocritical, and $\left(A_{j} x_{j}+I_{m_{t}}\right) \cap I_{j-1}=I_{m_{t}}$. One easily checks that $\sum_{j \in \Omega}\left[\left(A_{j} x_{j}+I_{m_{t}}\right) / I_{m_{t}}\right] \subseteq B / I_{m_{t}}$ is direct. Let $\Omega=$ $\left\{j_{1}, j_{2}, \cdots\right\}$. Set $M_{1}=B$, and for $u>1$ choose $M_{u}$ maximal with respect to $M_{u} \supseteqq \sum_{i=u}^{\infty}\left(A_{j_{i}} x_{j_{i}}+I_{m_{t}}\right), M_{u} \subseteq M_{u-1}$, and $M_{u} \cap\left(\sum_{i=1}^{u-1} A_{j_{i}} x_{j_{i}}+\right.$ $\left.I_{m_{t}}\right)=I_{m_{t}}$. Then then set $\left\{M_{u}\right\}_{u=1}^{\infty}$ forms a strictly descending chain of $\sigma$-closed submodules of $B$, which contradicts our assumption that $B$ has dec on $\sigma$-closed submodules. Hence $m_{t+1}$ exists.

Since $I_{m_{t}+1} / I_{m_{t}}$ is $\sigma$-cocritical, $V\left(I_{m_{t}+1} / I_{m_{t}}\right)=0$; so for each $t>0$, $m_{t+1} \geqq m_{t}+1>m_{t}$. Hence the sequence $\left\{m_{t}\right\}_{t=1}^{\infty}$ is strictly increasing and infinite. By Lemma 1.1 there exists a positive integer $n$ such that $V^{n+q} / V^{n+q+1} \in \mathscr{S}_{\sigma}$ for all $q \geqq 0$. Let $x \in I_{m_{n}+1}-I_{m_{n}}$. Then $V x \nsubseteq I_{m_{n-1}}$; thus we have $v_{1} x \notin I_{m_{n-1}}$ for some $v_{1} \in V$. But $V v_{1} x \nsubseteq$ $I_{m_{n-2}}$. So we inductively obtain $v_{2}, v_{3}, \cdots, v_{n-1} \in V$ such that $V v_{n-i} \cdots v_{2} v_{1} x \nsubseteq I_{m_{n-i}}$ for each $i=1,2, \cdots, n-1$. In particular, $V v_{n-1} v_{n-2} \cdots v_{2} v_{1} x \nsubseteq I_{m_{0}}=I_{0}$; hence $V^{n} x \nsubseteq I_{0}$. However, since $x \in$ $I_{m_{n}+1}$, we have that $V^{m_{n}+1} x \leqq I_{0}$ as $I_{w} / I_{w-1}$ is $\sigma$-cocritical for all $w \geqq 1$. It follows that there exists an integer $d \geqq n$ such that $V^{d} x \nsubseteq I_{0}$, but $V^{d+1} x \subseteq I_{0}$.

Now $\left(R x+I_{0} / I_{0}\right)$ is the homomorphic image of $R / V^{d+1}$ via $r+$ $V^{d+1} \stackrel{\alpha}{\rightarrow} r x+I_{0}$. We note that $0 \neq \alpha\left(V^{d} / V^{d+1}\right) \subseteq B / I_{0} \in \mathscr{F}_{o}$. However, since $d \geqq n, \alpha\left(V^{d} / V^{d+1}\right) \in \mathscr{T}_{\sigma}$. This contradicts the fact that $\mathscr{T}_{\sigma} \cap$ $\mathscr{F}_{o}=0$. Hence $I_{s}=B$ for some $s$ as desired.

2. Finitely generated injective modules in $F_{\sigma}$. In this section we study the relationship of finiteness conditions on injective hulls of cyclic modules and the dcc on $\sigma$-closed left ideals, where $\mathscr{L}_{\sigma}$ is exact. We obtain generalizations of several results of Faith and Walker [1].

A module $M$ is called $\sigma$-finitely generated if $M$ has a finitely generated submodule $N$ such that $M / N \in \mathscr{T}_{\sigma}$. Any finitely generated module is $\sigma$-finitely generated.

We use $E(M)$ to denote the injective hull of a module $M$, and we let $\phi_{M}$ be the natural homomorphism from $M$ into $\mathscr{L}_{\sigma}(M)$ (see [2], [4], [13] or [14]). If $\sigma$ is perfect, then the correspondence $K \rightarrow \mathscr{L}_{\sigma}(K)$ gives a lattice isomorphism from the lattice of $\sigma$-closed submodules $K$ of $M$ to the lattice of $Q_{\sigma}$-submodules of submodules of $\mathscr{L}_{\sigma}(M)$; the inverse isomorphism is given by $X \rightarrow \phi_{M}^{-1}(X)$ for each $Q_{\sigma}$-submodule $X$ of $\mathscr{L}_{\sigma}(M)$ - see [2], [4], [13] or [14]. If $\mathscr{L}_{\sigma}$ is exact and $R$ has acc on $\sigma$-closed left ideals, then $Q_{\sigma}$ must be a left noetherian ring by this lattice isomorphism (with $R=M$ ), and thus $Q_{\sigma}$ will contain a maximal two-sided nilpotent ideal $N$.

TheOREM 2.1. Let $\mathscr{L}_{\sigma}$ be exact, and let $R$ have acc on $\sigma$-closed 
left ideals. Let $N$ be the maximal nilpotent ideal of $Q_{\sigma}$. If $E\left(R / \phi_{R}^{-1}(N)\right)$ is $\sigma$-finitely generated, then $R$ has dcc on $\sigma$-closed left ideals.

Proof. Let $N^{\prime}=\phi_{R}^{-1}(N)$. Then $N^{\prime}$ is a nilpotent, two-sided ideal of $R$; since $\sigma$ is perfect, $N^{\prime}$ is also $\sigma$-closed and $N=\mathscr{L}_{\sigma}\left(N^{\prime}\right)=$ $Q_{\sigma} \otimes_{R} N$. Let $J$ be the injective hull of $Q_{\sigma} / N$ as a $Q_{\sigma}$-module. Since $\sigma$ is perfect, we have

$$
R / N^{\prime} \stackrel{\phi_{R / N^{\prime}}}{\longrightarrow} Q_{\sigma} \bigotimes_{R}\left(R / N^{\prime}\right) \cong Q_{o} / Q_{\sigma} \bigotimes_{R} N^{\prime}=Q_{\sigma} / N \cong J \cong E\left(R / N^{\prime}\right) .
$$

Since $E\left(R / N^{\prime}\right)$ is $\sigma$-finitely generated by hypothesis, then $E\left(R / N^{\prime}\right)$ has acc on $\sigma$-closed submodules by [9, Proposition 3.20]. Since $\sigma$ is perfect, then $E\left(R / N^{\prime}\right) / J \in \mathscr{F}_{\sigma}$ by [2, Proposition 17.1], hence $J$ also has acc on $\sigma$-closed $R$-submodules. Since every $Q_{o}$-submodule of $J$ is $\sigma$-closed as an $R$-submodule of $J$ (as $\sigma$ is perfect) then $J$ has acc on $Q_{\sigma}$-submodules. Consequently $J$ is finitely generated as a $Q$-module. By [1, Theorem 2.2] $Q_{\sigma}$ is a left artinian ring. Thus $R$ has dec on $\sigma$-closed left ideals via the lattice isomorphism between the lattice of $\sigma$-closed left ideals of $R$ and the lattice of left ideals of $Q_{\sigma}$.

Corollary 2.2. Let $\mathscr{L}_{\sigma}$ be exact, and let $R$ have acc on $\sigma$-closed left ideals. If injective hulls of cyclic modules in $\mathscr{F}_{0}$ are finitely generated, then $R$ has dcc on $\sigma$-closed left ideals.

It is now easy to obtain the main result of this section.

THEOREM 2.3. Let $\mathscr{L}_{\sigma}$ be exact. If each module in $\mathscr{F}_{\sigma}$ is contained in a direct sum of finitely generated modules, then $R$ has dec on $\sigma$-closed left ideals.

Proof. By [15, Theorem 1.2] $R$ has acc on $\sigma$-closed left ideals. Let $E$ be the injective hull of a cyclic module in $\mathscr{F}_{\sigma}$. By hypothesis, $E$ is contained in a direct sum of finitely generated modules; so $E$ is finitely generated by [1, Proposition 2.4]. The result now follows from Corollary 2.3.

3. A generalization of quasi-Frobenius rings. A ring is called quasi-Frobenius (QF) if it is both left and right artinian and left self-injective. A well-known theorem of Faith and Walker [1] states that a ring is $\mathrm{QF}$ if and only if every injective module is projective. It is also known [11, page 37] that $R$ is QF if and only if $R$ is left artinian (or noetherian) and $R$ is a cogenerator of $R$-mod. In this section we generalize these results. 
We call a module $W$ an $\mathscr{F}_{\sigma}$-cogenerator if every member of $\mathscr{F}_{\sigma}$ can be embedded in a product of copies of $W$. Following [10], [12], and their references, we say that a module $C$ is $\sigma$-codivisible if and only if $\operatorname{Ext}_{R}^{1}(C, F)=0$ for every $F \in \mathscr{F}_{\sigma}$. By [12, Theorem 8] a module $C$ is $\sigma$-codivisible if and only if $C / \sigma(R) C$ is a projective $R / \sigma(R)$-module.

Proposition 3.1. If every injective module in $\mathscr{F}_{\sigma}$ is $\sigma$-codivisible (projective), then $R$ has acc on $\sigma$-closed left ideals and $R / \sigma(R)$ $(R)$ is an $\mathscr{F}_{0}$-cogenerator.

Proof. Let $M \in \mathscr{F}_{\sigma}$ be injective. By assumption $M$ is projective as an $R / \sigma(R)$-module ( $R$-module). Thus $M$ is a direct summand of a direct sum of countably generated modules. By Kaplansky's theorem [7] $M$ is a direct sum of countably generated modules. Hence $R$ has acc on $\sigma$-closed left ideals by [15, Theorem 1.2].

Now let $N \in \mathscr{F}_{\sigma}$. Then $E(N)$ is $\sigma$-codivisible (projective) by hypothesis, which implies that $N$ is contained in a direct sum of copies of $R / \sigma(R)(R)$. So $R / \sigma(R)(R)$ is an $\mathscr{F}_{o}$-cogenerator.

Proposition 3.2. If $R$ has dec on $\sigma$-closed left ideals and $R / \sigma(R)$ is an $\mathscr{F}_{\sigma}$-cogenerator, then every injective module in $\mathscr{F}_{\sigma}$ is codivisible.

Proof. By Theorem $1.4 R$ has acc on $\sigma$-closed left ideals. Let $M \in \mathscr{F}_{\sigma}$ be injective. By [15, Theorem 1.2] $M$ is a direct sum of indecomposible modules. Thus we may assume that $M$ is indecomposible (as a direct sum of $\sigma$-codivisible modules is $\sigma$-codivisible). Since $M \in \mathscr{F}_{\sigma}$ and $R$ has dec on $\sigma$-closed left ideals, $M$ contains a $\sigma$ cocritical submodule $N$. By assumption $M=E(N)$ is embedded in a direct product $U$ of copies of $R / \sigma(R)$. Choose a projection map $p: U \rightarrow R / \sigma(R)$ such that $p(N) \neq 0$. We see that the restriction of $p$ to $N$ is one-to-one as $N$ is $\sigma$-cocritical and $R / \sigma(R) \in \mathscr{F}_{\sigma}$. Since $N$ is essential in $M, p$ must also be one-to-one on $M$. Consequently, $M$ is isomorphic to a direct summand $p(M)$ of $R / \sigma(R)$; this implies $M$ is $\sigma$-codivisible since $R / \sigma(R)$ is.

Let $W \in \mathscr{F}_{\sigma}$ be an injective module that cogenerates the torsionfree class $\mathscr{F}_{\sigma}$. Using a proof similar to the one just given, one easily shows that if $R$ has dec on $\sigma$-closed left ideals and $W$ is $\sigma$ codivisible (projective), then every injective module in $\mathscr{F}_{\sigma}$ is $\sigma$ codivisible (projective).

COROLlary 3.3. $R$ has dec on $\sigma$-closed left ideals if and only if every injective module in $\mathscr{F}_{\sigma}$ is a direct sum of injective hulls of $\sigma$-cocritical modules. 
Proof. The proof of the "only if" part is contained in the proof of Proposition 3.2.

Suppose that $I_{1} \supseteqq I_{2} \supseteqq \cdots$ is a descending chain of $\sigma$-closed left ideals of $R$, and let $I=\bigcap_{n=1}^{\infty} I_{n}$. Since $R / I \in \mathscr{F}_{\sigma}$ is cyclic, it follows from the hypothesis that $E(R / I)$ contains a finite, essential, direct sum $M$ of $\sigma$-cocritical submodules. By [5, Corollary 1.5] $M \cap(R / I)$ has $\sigma$-finite length.

We claim that

$$
\left({ }^{* * *}\right) \quad M \cap(R / I) \cap\left(I_{1} / I\right) \supseteqq M \cap(R / I) \cap\left(I_{2} / I\right) \supseteqq \cdots
$$

is a descending chain of $\sigma$-closed submodules of $M \cap(R / I)$. To see this, let $f_{j}$ be the natural composition

$$
(R / I) / M \cap\left(I_{j} / I\right) \longrightarrow(R / I) /\left(I_{j} / I\right) \longrightarrow R / I_{j} .
$$

Let $g_{j}$ be the restriction of $f_{j}$ to $(M \cap(R / I)) /\left(M \cap\left(I_{j} / I\right)\right)$. Then ker $g_{j}=$ $\operatorname{ker} f_{j} \cap\left[M /\left(M \cap\left(I_{j} / I\right)\right)\right]=\left[\left(I_{j} / I\right) /\left(M \cap\left(I_{j} / I\right)\right)\right] \cap\left[M /\left(M \cap\left(I_{j} / I\right)\right)\right]=0$; so $g_{j}$ is a monomorphism into $R / I_{j} \in \mathscr{F}_{\sigma}$. Hence $(M \cap(R / I)) /\left(M \cap\left(I_{j} / I\right)\right) \in$ $\mathscr{F}_{\sigma}$ for each $j$.

By [5, Proposition 1.2] the chain $\left(^{* * *}\right)$ must terminate. Since $\bigcap_{n=1}^{\infty}\left(I_{n} / I\right)=0$, then there exists a positive integer $k$ such that $M \cap(R / I) \cap\left(I_{k} / I\right)=0$. Since $M$ is essential in $E(R / I)$, then $(R / I) \cap$ $\left(I_{k} / I\right)=0$, and hence $I_{k}=I$. Therefore, the chain $I_{1} \supseteqq I_{2} \supseteqq \cdots$ terminates.

As usual, we call the torsion class $\mathscr{T}_{\sigma}$ a TTF class if $\mathscr{T}_{\sigma}$ is closed under direct products. If $\mathscr{T}_{\sigma}$ is a TTF class, then there exists a (necessarily unique and idempotent) ideal $T$ in the filter $F\left(\mathscr{T}_{\sigma}\right)=$ $\left\{I \mid R / I \in \mathscr{F}_{\sigma}\right\}$. If $N$ is a $\sigma$-cocritical module in $\mathscr{F}_{\sigma}$, then $T N$ is a simple module. Indeed, $T N \neq 0$ since $N \in \mathscr{F}_{\sigma}$; and if $K$ is a nonzero submodule of $T N$, we must have $T N / K=T(T N / K)=0$ as $T N$ is $\sigma$ cocritical. Thus in the TTF case we have the following result.

Corollary 3.4. Let $\mathscr{T}_{\sigma}$ be a TTF class. Then $R$ has dec on $\sigma$-closed left ideals if and only if every injective module in $\mathscr{F}_{\sigma}$ is a direct sum of injective envelopes of simple modules.

In case $\mathscr{L}_{\sigma}$ is exact, we can strengthen Propositions 3.1 and 3.2 considerably.

THEOREM 3.5. If $\mathscr{L}_{\sigma}$ is exact, then the following statements are equivalent:

(1) $R$ has dec on $\sigma$-closed left ideals, and $R / \sigma(R)$ is an $\mathscr{F}_{\sigma^{-}}$ cogenerator.

(2) $R$ has acc on $\sigma$-closed left ideals, and $R / \sigma(R)$ is an $\mathscr{F}_{\sigma^{-}}$ cogenerator. 
(3) Every injective module in $\mathscr{F}_{\sigma}$ is $\sigma$-codivisible.

(4) $R$ has dec on $\sigma$-closed left ideals, and any injective $\mathscr{F}_{\sigma^{-}}$ cogenerator in $\mathscr{F}_{\sigma}$ is $\sigma$-codivisible.

(5) $R$ has acc on $\sigma$-closed left ideals, and any injective $\mathscr{F}_{\sigma^{-}}$ cogenerator in $\mathscr{F}_{\sigma}$ is $\sigma$-codivisible.

Furthermore, any of these five equivalent statements imply that $Q_{\sigma}$ is a $\mathrm{QF}$ ring.

REMARK. In analogy with $\mathrm{QF}$ rings, one might expect to find that $R / \sigma(R)$ is $\sigma$-injective (that is, $R / \sigma(R)=Q_{\sigma}$ ) and hence that $R / \sigma(R)$ is QF when the hypotheses of Theorem 3.5 are satisfied. However, it is trivial to give examples where this is not the case. In particular, let $R$ be then $2 \times 2$ upper triangular matrix ring over a field $F$, and let $\mathscr{T}_{\sigma}$ be the class of all modules annihilated by the top row of $R$; then $R$ and $\sigma$ satisfy the hypotheses of Theorem 3.5, $R=R / \sigma(R)$ is not $\mathrm{QF}$, and $Q_{o}$ is the full $2 \times 2$ matrix ring over $F$.

Proof of 3.5. That (1) implies (3) is Proposition 3.2. That (3) implies (2) follows by Proposition 3.1.

If (2) holds, then $\sigma$ is perfect, and hence $Q_{\sigma}$ is left noatherian via (2). We claim that $Q_{\sigma}$ is a cogenerator in the category $Q_{\sigma}$-mod of unital left $Q_{\sigma}$-modules. Since any left $Q_{\sigma}$-module $M$ is in $\mathscr{F}_{\sigma}$ when viewed as an $R$-module, then $M$ is embedded in a direct product of copies of $R / \sigma(R)$. Thus there is an $R / \sigma(R)$-monomorphism $\alpha: M \rightarrow N$, where $N$ is a direct product of copies of $Q_{\sigma}$. Let $q \in Q_{\sigma}$, let $m \in M$, and consider $(q m) \alpha-q((m) \alpha)$. Since $Q_{\sigma} /(R / \sigma(R)) \in \mathscr{T}_{\sigma}$, there is a left ideal $K \in F\left(\mathscr{T}_{\sigma}\right)=\left\{I \mid R / I \in \mathscr{T}_{\sigma}\right\}$ such that $K q \subseteq R / \sigma(R)$. Now for any $k \in K$, we have $k((q m) \alpha-q((m) \alpha))=k(q m) \alpha-k q((m) \alpha)=(k q m) \alpha-$ $(k q m) \alpha=0$. Hence $\alpha$ is a $Q_{\sigma}$-monomorphism; that is, $Q_{o}$ is a cogenerator for $Q_{\sigma}$-mod. Consequently, $Q_{\sigma}$ is a $Q F$ ring [11, page 373]; so $Q_{\sigma}$ is left artinian. Since $\sigma$ is perfect, it follows that $R$ has dec on $\sigma$-closed left ideals, and (1) follows.

$(3) \Rightarrow(4)$. In view of $(3)$, any injective $\mathscr{F}_{\sigma}$-cogenerator is certainly codivisible. Moreover, $R$ has dec on $\sigma$-closed left ideals since we have shown that (3) implies (1).

That (4) implies (5) follows from Theorem 1.4.

$(5) \Rightarrow(2)$. Let $W \in \mathscr{F}_{\sigma}$ be an injective $\mathscr{F}_{\sigma}$-cogenerator. Since $W$ is $\sigma$-codivisible by (5), then $W$ is a direct summand of a direct sum of copies of $R / \sigma(R)$; hence $R / \sigma(R)$ must also be an $\mathscr{F}_{\sigma}$-cogenerator.

Theorem 3.6. Assume that (i) $R$ has dec on $\sigma$-closed left ideals, (ii) $R$ is an $\mathscr{F}_{0}$-cogenerator, and (iii) if $M$ contains an essential $\sigma$ cocritical submodule $N$ which is isomorphic to a submodule of a direct product of copies of $\sigma(R)$, then $M$ is isomorphic to a submodule 
of a projective module. Then every injective module in $\mathscr{F}_{\sigma}$ is projective. The converse is true if $\mathscr{L}_{\sigma}$ is exact.

Proof. Let $M \in \mathscr{F}_{\sigma}$ be an injective module. As in the proof of Proposition 3.2, we may assume that $M=E(N)$, where $N$ is $\sigma$ cocritical. By (ii) $M$ is embedded in a direct product $U$ of copies of $R$. If there is no projection map $p: U \rightarrow R$ such that $p(N) \nsubseteq \sigma(R)$, then $N$ is embedded in a direct product of copies of $\sigma(R)$. Thus by (iii) $M$ is isomorphic to a submodule of a projective module; this implies that $M$ is projective, as $M$ is given to be injective. Now assume that there is a projection map $p: U \rightarrow R$ such that $p(N) \nsubseteq \sigma(R)$. Then the restriction of $p$ to $N$ is one-to-one, as $N$ is $\sigma$-cocritical. Since $N$ is essential in $M$, we also have that $p$ is one-to-one on $M$. Consequently $M$ is projective as it is isomorphic to a direct summand of $R$.

For the converse assume that $\mathscr{L}_{\sigma}$ is exact and that every injective module in $\mathscr{F}_{\sigma}$ is projective. By Proposition $3.1, R$ is an $\mathscr{F}_{\sigma^{-}}$ cogenerator. By assumption every module in $\mathscr{F}_{\sigma}$ is contained in a projective module, namely its injective hull. Thus (iii) holds trivially, and (i) holds by Theorem 2.4.

REMARKS. We note that conditions (i), (ii), and (iii) are independent; that is, there exist $\sigma$ such that $\mathscr{L}_{\sigma}$ is exact and any two of (i), (ii) or (iii) hold while the remaining condition fails. Moreover, each of the following conditions is sufficient to imply condition (iii) of Proposition 3.6.

(1) For each $\sigma$-cocritical module $N, \operatorname{Hom}_{R}(N, \sigma(R))=0$.

(2) $\mathscr{T}_{\sigma}$ is a TTF class.

(3) $Z(R) \cap \sigma(R)=0$, where $Z(R)$ denotes the singular submodule of $R$.

(4) $\sigma(R)$ contains no nilpotent ideals of $R$.

As a question related to the ideas in this paper, one might ask whether every injective module in $\mathscr{F}_{\sigma}$ being $\sigma$-codivisible is equivalent to every $\sigma$-codivisible module in $\mathscr{F}_{\sigma}$ being injective. We easily resolve this question in our closing result.

Proposition 3.7. The following statements are equivalent.

(1) Every $\sigma$-codivisible module in $\mathscr{F}_{\sigma}$ is injective.

(2) $R / \sigma(R)$ is a $\mathrm{QF}$ ring.

(3) Every injective module in $\mathscr{F}_{\sigma}$ is $\sigma$-codivisible, and $\mathscr{F}_{\sigma}$ is closed under homomorphic images.

Proof. $(1) \Longrightarrow(2)$. Let $X$ be a projective $R / \sigma(R)$-module. As an $R$-module, $X \in \mathscr{F}_{\sigma}$, and $X$ is $\sigma$-codivisible by [12, Theorem 8]. By 
(1) $X$ is injective as an $R$-module, and hence $X$ is also injective as an $R / \sigma(R)$-module. That every projective $R / \sigma(R)$-module is injective is well-known to imply that $R / \sigma(R)$ is $\mathrm{QF}$.

$(2) \Longrightarrow(1)$. Let $X \in \mathscr{F}_{\sigma}$ be $\sigma$-codivisible. Then $X$ is projective as an $R / \sigma(R)$-module by [12, Theorem 8]. Hence $X$ is an injective $R / \sigma(R)$-module since $R / \sigma(R)$ is $\mathrm{QF}$ by assumption. Since $X \in \mathscr{F}_{\sigma}$, then $X$ is also injective as an $R$-module by [9, Proposition 4.8].

(2) $\Rightarrow(3)$. If $M \in \mathscr{F}_{\sigma}$ is injective, then $M$ is also injective as an $R / \sigma(R)$-module. Hence $M$ is a projective $R / \sigma(R)$-module by (2). This implies $M$ is $\sigma$-codivisible by [12, Theorem 8].

If $Y$ is an $R$-homomorphic image of $M \in \mathscr{F}_{o}$, then $Y$ is also an $R / \sigma(R)$-module as $\sigma(R) M=0$. However, $R / \sigma(R)$ is a cogenerator for $R / \sigma(R)-\bmod$ by $(2)$, which implies that $Y \subseteq \Pi R / \sigma(R)$. Hence $Y \in \mathscr{F}_{\sigma}$.

$(3) \Rightarrow(2)$. Let $M$ be injective as an $R / \sigma(R)$-module. Since $\mathscr{F}_{\sigma}$ is closed under homomorphic images, every $R / \sigma(R)$-module when viewed as an $R$-module is in $\mathscr{F}_{\sigma}$. Thus by [9, Proposition 4.8] $M$ is injective as an $R$-module. By assumption $M$ is $\sigma$-codivisible; and therefore, as an $R / \sigma(R)$-module $M$ is projective [12, Theorem 8]. Thus $R / \sigma(R)$ is $\mathrm{QF}$ as every injective $R / \sigma(R)$-module is projective.

\section{REFERENCES}

1. C. Faith and E. A. Walker, Direct sum representations of injective modules, J. Algebra, 5 (1967), 203-221.

2. J. S. Golan, Localization of Noncommutative Rings, Pure and Applied Math. 30, Marcel Dekker, New York, 1975.

3. - Modules satisfying both chain conditions with respect to a torsion theory, Proc. Amer. Math. Soc., 52 (1975), 103-108.

4. O. Goldman, Rings and modules of quotients, J. Algebra, 13 (1969), 10-47.

5. - Elements of noncommutative arithmetic 1, J. Algebra, 35 (1965), 308-341.

6. C. Hopkins, Rings with minimal condition for left ideals, Ann. Math., 40 (1939), 712-730.

7. I. Kaplansky, Projective modules, Ann. Math., 68 (1958), 372-377.

8. J. Levitzki, On rings which satisfy the minimum condition for right-hand ideals, Compositio Math., 7 (1939), 214-222.

9. J. Manocha, Finiteness conditions and torsion theories, Thesis, University of Wisconsin, 1973.

10. R. Miller and M. L. Teply, On flatness relative to a torsion theory, Commun. in Algebra, (to appear).

11. B. L. Osofsky, A generalization of quasi-Frobenius rings, J. Algebra, 4 (1966), 373-387.

12. K. M. Rangaswamy, Codivisible modules, Commun. in Algebra, 2 (1974), 475-489.

13. B. Stenström, Rings and Modules of Quotients, Lecture Notes in Math. 237, Springer-Verlag, Berlin, 1971.

14. - Rings of Quotients, Die Grundlehren der Math. Wissen. in Einzeldarstellungen 217, Springer-Verlag, Berlin, 1975.

15. M. L. Teply, Torsion-free injective modules, Pacific J. Math., 28 (1969), 441-453.

Received August 31, 1977 and in revised form September 21, 1977. The first author 
was supported by the National Science Foundation under grant MCS 77-01818. The second author was supported by a Faculty Research Grant at the College of William and Mary.

College of William and Mary

WILLIAMSBURG, VA 23185

AND

UNIVERSITY OF FLORIDA

GaINESVILLe, FL 32611 



\section{PACIFIC JOURNAL OF MATHEMATICS}

EDITORS

DONALD BABBITT (Managing Editor)

University of California

Los Angeles, California 90024

Hugo RossI

University of Utah

Salt Lake City, UT 84112

C. C. MOORE and ANDREW OGG

University of California

Berkeley, CA 94720

\section{J. DUGUNDJI}

Department of Mathematics University of Southern California Los Angeles, California 90007

R. Finn and J. Milgram Stanford University

Stanford, California 94305

\section{ASSOCIATE EDITORS}
E. F. BECKENBACH
B. H. Neumann
F. WOLF
K. YoSHIDA

\section{SUPPORTING INSTITUTIONS}

UNIVERSITY OF BRITISH COLUMBIA

CALIFORNIA INSTITUTE OF TECHNOLOGY

UNIVERSITY OF CALIFORNIA

MONTANA STATE UNIVERSITY

UNIVERSITY OF NEVADA, RENO

NEW MEXICO STATE UNIVERSITY

OREGON STATE UNIVERSITY

UNIVERSITY OF OREGON
UNIVERSITY OF SOUTHERN CALIFORNIA

STANFORD UNIVERSITY

UNIVERSITY OF HAWAII

UNIVERSITY OF TOKYO

UNIVERSITY OF UTAH

WASHINGTON STATE UNIVERSITY

UNIVERSITY OF WASHINGTON 


\section{Pacific Journal of Mathematics}

\section{Vol. 83, No. 1 \\ March, 1979}

Richard Neal Ball, Topological lattice-ordered groups ............... 1

Stephen Berman, On the low-dimensional cohomology of some

infinite-dimensional simple Lie algebras .................. 27

R. P. Boas and Gerald Thomas Cargo, Level sets of derivatives ......... 37

James K. Deveney and John Nelson Mordeson, Splitting and modularly

perfect fields......................................

Robert Hugh Gilman and Ronald Mark Solomon, Finite groups with small

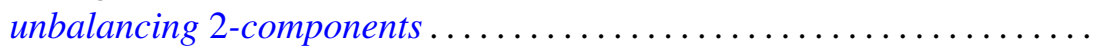

George Grätzer, Andras Hajnal and David C. Kelly, Chain conditions in free products of lattices with infinitary operations..................

Benjamin Rigler Halpern, Periodic points on tori ..................

Dean G. Hoffman and David Anthony Klarner, Sets of integers closed under

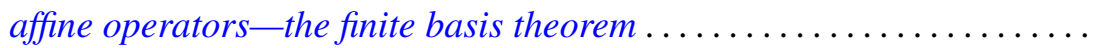

Rudolf-Eberhard Hoffmann, On the sobrification remainder ${ }^{s} X-X \ldots \ldots$

Gerald William Johnson and David Lee Skoug, Scale-invariant

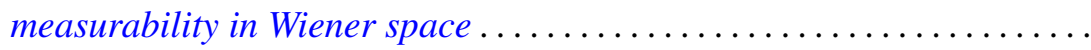

Michael Keisler, Integral representation for elements of the dual of

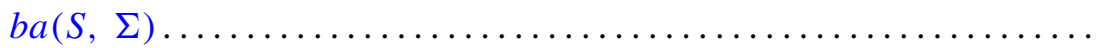

Wayne C. Bell and Michael Keisler, A characterization of the representable Lebesgue decomposition projections ................

Wadi Mahfoud, Comparison theorems for delay differential equations ...

R. Daniel Mauldin, The set of continuous nowhere differentiable functions .

Robert Wilmer Miller and Mark Lawrence Teply, The descending chain condition relative to a torsion theory...

Yoshiomi Nakagami and Colin Eric Sutherland, Takesaki's duality for regular extensions of von Neumann algebras ........ .

William Otis Nowell, Tubular neighborhoods of Hilbert cube manifolds ...

Mohan S. Putcha, Generalization of Lentin's theory of principal solutions of word equations in free semigroups to free product of copies of positive reals under addition

Amitai Regev, A primeness property for central polynomials . ...

Saburou Saitoh, The Rudin kernels on an arbitrary domain. . .

Heinrich Steinlein, Some abstract generalizations of the

Ljusternik-Schnirelmann-Borsuk covering theorem . . . 